RAL-TR-98-030

\title{
A HIGH RESOLUTION, IMAGING NEUTRON BEAM MONITOR USING GAS MICROSTRIP TECHNOLOGY
}

\author{
J E Bateman, N J Rhodes and R Stephenson \\ Rutherford Appleton Laboratory, Chilton, Didcot, OX11 0QX, U.K.
}

18 March 1998

\begin{abstract}
The performance of a new high resolution, position-sensitive beam monitor for slow neutrons on the ISIS spallation neutron source is reported. Based on gas microstrip technology developed at RAL for particle physics applications, the monitor delivers high spatial resolution $(\sigma \approx 0.1 \mathrm{~mm})$ combined with a fast timing response, high data rate capability and a low gamma sensitivity.
\end{abstract}




\section{INTRODUCTION}

Each of the 17 neutron scattering instruments at ISIS operates a number of beam line monitors to measure neutron beam intensities at various points in the beam lines. To date these have been scintillation monitors using GS20 glass. These have been very successful but are somewhat sensitive to gamma radiation and offer no information on the distribution of neutron intensity and energy within beam profiles.

There are now a number of areas where position sensitive beamline monitors would be advantageous. Transmission measurements of engineering samples can be analysed to provide structural information about the samples. Resonance radiography requires two dimensional monitor readout. Position sensitive beam line monitors will allow more accurate determination of corrections for sample absorption. They will permit collimation-defining beam line components to be more accurately placed, improving signal to noise ratios. Beam profiles of instruments incorporating neutron guides such as HRPD and IRIS are non-homogeneous with respect to energy. Position sensitive monitor readouts will allow these profiles to be accurately determined.

The lithographic gas microstrip detector (MSGD) was introduced by Anton Oed of the ILL and has been developed by him and his colleagues as a detector of scattered neutrons [1]. At RAL we have concentrated on the application of the MSGD in particle physics, and in particular on the aspects of high counting rates and long lifetimes demanded by application at the LHC at CERN. One outcome of this programme was a prototype beam tracking chamber which was tested successfully in high rate beams [2]. This device has an active area of $82 \mathrm{mmx} 60 \mathrm{~mm}$ defined by 272 strip sections of $0.3 \mathrm{~mm}$ width, each section being $60 \mathrm{~mm}$ high. The lithographic pattern of $10 \mu \mathrm{m}$ wide anodes interleaved with $90 \mu \mathrm{m}$ wide cathodes is produced in a gold process on Schott S8900 semiconducting glass. The active depth of the conversion space is $3 \mathrm{~mm}$.

Tests carried out on the PEARL beam line at ISIS confirmed that the attractive properties of the MSGD for applications in particle physics, namely sub-millimeter spatial resolution and high counting rate capability, are carried over into applications on neutron beam lines with the added advantage that the gas converter $\left({ }^{3} \mathrm{He}\right.$ plus a quencher) yields a very low gamma sensitivity [3]. In particular it was demonstrated that with the fine granularity (mm scale) permitted by the MSGD it is possible to achieve sub-millimeter spatial resolution provided (as is the case in a beam monitor) a high detection efficiency is not required. In order to further develop the MSGD for high resolution beam imaging, work was carried out on two fronts:

(i) A montecarlo model of the conversion process of the slow neutron in the MSGD (the determining factor in the spatial resolution) was developed and used to explore the envelope of design options (strip widths, conversion depth, gas fills, discriminator thresholds, etc) [4]. This work showed that if the intrinsic strip width $(0.3 \mathrm{~mm})$ of the existing MSGD were combined with a narrowed conversion depth $(1 \mathrm{~mm})$ and a moderately hyperbaric fill of 1.5 bar ${ }^{3} \mathrm{He}$ with 1.5 bar $\mathrm{CF}_{4}$ (i.e. two bars on the detector window) spatial resolution down to the level of $\sigma \leq 0.25 \mathrm{~mm}$ could be anticipated with a detection efficiency of around $1 \%$. 
(ii) The MSGD beam counter was redesigned so as to be more suitable for neutron beam work:

- The melinex windows were replaced by covers with $2 \mathrm{~mm}$ thick aluminium windows and "O" ring seals of sufficient strength to support hyperbaric operation and give long-term stable gas conditions. The detector was proofed to six bars for hyperbaric operation.

- The readout was arranged so that each $0.3 \mathrm{~mm}$ wide section was instrumented over a range of 48 channels (16mm).

- The drift electrode support system was modified to permit it to be located at one millimeter from the microstrip plate.

With these modifications a test was prepared on the ROTAX beamline on ISIS to explore the properties of the modified MSGD.

\section{THE ROTAX BEAM}

The ROTAX beam used for our tests is a parasitic line behind the PRISMA facility. A beam-chopper controls the neutron access to the line cutting out the highest energy neutrons and the gamma flash. The beam intensity and spectrum is highly variable being dependent on the conditions at the PRISMA target station (in principle a flux of up to a maximum of $\approx 10^{7} \mathrm{n} / \mathrm{cm}^{2} / \mathrm{s}$ is available). During the current test period the observed mean channel counting rates in the MSGD were typically $\approx 200 \mathrm{~Hz}$ and not capable of challenging the rate capability of the detector system. The neutron intensity at the sample positions is modulated on a $50 \mathrm{~Hz}$ cycle in synchronisation with the ISIS pulsed proton beam. Neutron counts from a detector or monitor are time stamped and histogrammed into time bins by the ISIS Data Acquisition Electronics (DAE). The widths of the time bins can be programmed in a variety of ways. The minimum bin width is $0.25 \mu \mathrm{s}$ which determines the time resolution requirement of the counter.

The MSGD was placed at the far end of the ROTAX beam line, approximately two meters beyond the ROTAX target chamber and four meters beyond the PRISMA beam monitor (scintillator) which was recorded with the MSGD data for reference purposes. The distance of the MSGD from the ISIS moderator was approximately 17 meters.

\section{DETECTOR AND READOUT ARRANGEMENT}

The particle physics beam chamber has a plate with an anode pitch of $0.3 \mathrm{~mm}$. By using 3 standard (16 channel) amplifier cards [2] 48 channels of readout are obtained giving an active width of $16 \mathrm{~mm}$. The active height of the counter (along the strips) is $60 \mathrm{~mm}$. The amplifiers give amplitudes of $\approx 4 \mathrm{mV} / \mathrm{keV}$ of energy deposit in the detector with a rise time of $\approx 50 \mathrm{~ns}$ (set by the detector) and a recovery time constant of $100 \mathrm{~ns}$. These signals are transported to a remote electronics rack via $12 \mathrm{~m}$ long coaxial cables and are received in LeCroy NIM Amplifier modules (Model 612) which provide a further x10 signal gain. 
For the discriminators we used 6 commercial 8-fold NIM units (NE 4684), the outputs of which were used to drive the inputs of 12 ISIS data input modules (DIM). The DIM modules constitute the front end of the ISIS DAE system. The signals from the standard scintillation beam monitor were also recorded via a spare DIM channel. At the discriminator inputs pulses of $>1 \mathrm{~V}$ were recorded at the maximum $\mathrm{V}_{\mathrm{c}}$ used with the $3 \mathrm{bar}$ gas filling and $>2 \mathrm{~V}$ with the $5.4 \mathrm{bar}$ gas filling. The discriminator thresholds were set to a uniform $300 \mathrm{mV}$.

For most of the tests the counter was filled with a gas mixture of $50 \%{ }^{3} \mathrm{He}$ and $50 \% \mathrm{CF}_{4}$ at 3 bar (absolute) total pressure. At the end of the session brief tests were carried out with approximately double this pressure (5.4bar). The HT biases applied to the counter were: $-2.5 \mathrm{kV}$ on the drift electrode and $-800 \mathrm{~V}$ to $-1000 \mathrm{~V}$ on the cathode strips for the 3 bar case (the anodes being at earth). In the 5.4bar case the cathode voltage range rose to $-1050 \mathrm{~V}$ to $-1300 \mathrm{~V}$. The gas filling rig permitted the counter volume to be pumped out before filling, thus facilitating leak testing and also guaranteeing the purity of the gas.

In a detector array in which a physical event can simultaneously trigger adjacent channels, the differential linearity is a sensitive function of the gain uniformity of the individual channels. Measurement during the previous tests showed that the physics could amplify the rms gain spread in the electronic channels by a factor of 3.4 [3]. The introduction of the extra factor of ten in gain and the increased energy deposit in the high pressure gas reduced the sensitivity of the system to this effect and permitted a gain uniformity of around $4 \%$ with a common threshold and no channel by channel calibration.

Calibration of the energy equivalent of the discriminator threshold (LLD) by means of an x-ray induced signal was not possible due to the thick window and low X-ray stopping power of the gas. It was therefore necessary to fall back on the pulse height spectrum predicted by the Montecarlo model for this purpose as described below.

\section{RESULTS}

\section{(i) Beam Imaging}

Figure 1 shows the cross-section of the ROTAX beam as imaged by the MSGD at $\mathrm{V}_{\mathrm{c}}=-$ $1000 \mathrm{~V}$. The usual gaussian core is observed with clipping at the right hand side due to the intervention of beam optics on this side. The $\sigma$ of the gaussian is $7.56 \mathrm{~mm}$ giving a FWHM of $17.8 \mathrm{~mm}$ for the beam. The RMS of the residuals of the fit of the channel counts to the gaussian profile is $4.4 \%$ which is quite acceptable considering that no channel alignment has been performed. Experience with the previous test showed that it is quite straightforward to reduce the RMS differential non-linearity to around $0.5 \%$ with the appropriate calibration techniques [3].

\section{(ii) Time Spectrum}

Figure 2 shows the flight time spectrum of the neutron events recorded in figure 1 . The time bin is $0.5 \mu \mathrm{s}$. The MSGD spectrum contains five times more counts than that of the 
PRISMA beam monitor (recorded simultaneously) but otherwise shows the same time structure when allowance is made for the $4 \mathrm{~m}$ difference in flight path.

(iii) Pulse Height and Gain Calibration

Histogramming the pulses from one of the MSGD strips results in a distribution of the form shown in figure 3. In this case (using the 5.4bar gas fill) a distinct peak is observed in the pulse height distribution (as is also the case with the 3bar filling). Also shown in figure 3 is the distribution of energy deposits in a detector strip predicted by the Montecarlo model with the parameters set to match the experimental conditions. Normalising the two distributions together gives a very convincing fit and permits the calibration of the pulse heights in terms of the energy deposit. The peaks occur at $100 \mathrm{keV}$ in the 3 bar filling and $210 \mathrm{keV}$ in the 5.4 bar filling, i.e. approximately scaling with the pressure.

This calibration has two very useful spin-offs - first, in conjunction with the use of the charge calibration test pulse (injected into every channel) it permits an approximate estimation of the gas gain of the counter. If one assumes a mean energy per ion pair in the gas of $28 \mathrm{eV}$ the gain curves of figure 4 result. The poor point on the $3 \mathrm{bar}$ curve results from the difficulty of resolving the peak from the noise at the lowest gain. The second benefit of the pulse height calibration is that, once the gain is known the effective energy of the LLD setting on the discriminators can be deduced and the energy LLD varied by changing $\mathrm{V}_{\mathrm{c}}$ rather than the electronic thresholds which had no common control and could only be changed laboriously.

\section{(iv) Spatial Resolution}

The spatial resolution was measured by means of a slit made by separating two $10 \mathrm{~mm}$ thick sheets of sintered boron carbide with a $0.25 \mathrm{~mm}$ shim. Because of their size the sheets could not be placed closer than $50 \mathrm{~mm}$ to the front of the detector. Figure 5 shows the response of the detector system to this collimated beam with the detector moved $4.5 \mathrm{~mm}$ between exposures. In one case the slit is lined up with a detector strip and in the other it overlaps two strips. In both cases the point spread function (PSF) fits very well to a double gaussian with $63 \%$ of the signal contained in the narrower peak with $\sigma=0.17 \mathrm{~mm}$ and $37 \%$ in the wider peak with $\sigma=0.84 \mathrm{~mm}$ (averages). The cathode potential is $-850 \mathrm{~V}$ (3bar filling) corresponding to an energy LLD of $234 \mathrm{keV}$. (This is raw data; the width of the $0.25 \mathrm{~mm}$ slit has not been removed.)

The spatial resolution was investigated systematically as a function of the (energy) LLD by varying $\mathrm{V}_{\mathrm{c}}$ in steps of $50 \mathrm{~V}$ from $-800 \mathrm{~V}$ to $-1000 \mathrm{~V}$. As figure 4 shows, this corresponds to a gain multiplier of approximately 1.75 per $50 \mathrm{~V}$ step with the energy threshold moving in an inverse manner from $402 \mathrm{keV}$ down to $44.5 \mathrm{keV}$. Figure 6 shows the measured PSFs area normalised and figure 7 summarises the parameters of the double gaussian fits. At $L L D=402 \mathrm{keV} 62 \%$ of the signal lies in a gaussian with $\sigma=0.12 \mathrm{~mm}$ and the rest in one with $\sigma=0.48 \mathrm{~mm}$. This spatial resolution is achieved at the cost of a severe reduction in sensitivity. 


\section{(v) Sensitivity}

The sensitivity of the MSGD was measured in terms of kilocounts in the raw PSFs of figure 6 per $\mu \mathrm{Ah}$ of ISIS beam current. Figure 7 shows this parameter as a function of the energy LLD. Calibration of the sensitivity in terms of a known detection efficiency was arranged by comparing the counting rate observed in the MSGD in a collimated section of beam $4 \mathrm{~mm}$ wide by $50 \mathrm{~mm}$ high with that observed in a lithium glass scintillation monitor aligned with the same portion of beam. Using the known efficiency of the scintillator at $1 \mathrm{~A}$ the comparison indicated a sensitivity of $4.8 \%$ for the MSGD.

Because of multiple detection of events in the MSGD the sensitivity of the detector can be typically higher than the nominal conversion efficiency of $1.2 \%$. Figure 8 shows the sensitivity predicted by the Montecarlo model as a function of energy LLD. At $L L D=50 \mathrm{keV}$ a sensitivity of $3.5 \%$ is indicated. Given the uncertainties of the calibration process (e.g. the whole energy spectrum was used to get good statistics) this is reasonable agreement. Normalising the measured sensitivity to the Montecarlo at $L L D=50 \mathrm{keV}$ results in a very satisfactory agreement between the predicted and observed sensitivity curves.

(vi) Spatial resolution with 5.4bar gas filling

The sequence of PSFs versus $V_{c}$ (i.e. energy LLD) was repeated briefly with the MSGD filled with the gas mixture at 5.4bar absolute. The resulting PSFs (area-normalised) are presented in figure 9 .

\section{DISCUSSION}

\section{(i) Beam Imaging}

No attempt was made during these tests to set up the hardware to optimise the differential linearity; hence the $4.4 \%$ RMS non-linearity seen in figure 1 . This effect degrades at higher LLD settings $(12.2 \%$ at $\mathrm{LLD}=234 \mathrm{keV})$ indicating that this procedure is essential for application of the detector as a beam monitor. The experience of the previous test [3] showed that it was quite simple to achieve 2\% RMS in the hardware and $0.6 \%$ RMS with an off-line software correction. The considerably improved pulse height spectrum of the hyperbaric detector (relative to the situation in [3]) implies that the necessary calibration will be simple and effective.

The high spatial resolution of the MSGD clearly has advantages in the monitoring and setting up of a relatively small beam such as the ROTAX one, the FWHM of $17.8 \mathrm{~mm}$ occupying 59 detector channels.

Due to the conditions of the present test period it was not practicable to study the medium to long term stability of the MSGD as a beam monitor. However, the short term stability over the two days of testing appeared to be excellent with no detectable shift in the pulse height spectrum. The fact that the detector is now a sealed unit with rigid walls decouples the gas gain from ambient conditions. 
The low neutron flux of the ROTAX beam precluded any significant test of the rate capabilities of the MSGD. However, these are so well verified in the particle physics application [2] that this is not a concern.

\section{(ii) The Time Spectrum}

The time spectra of figure 2 do not reveal the particular advantage of the MSGD in respect of its insensitivity to gamma rays since the chopper cuts off the gamma flash. While the gamma stopping power of the present detector is increased (relative to that of the detector in [3]) the stopping power for the triton and proton scales similarly, as do the discriminator thresholds. Consequently, no change in the gamma insensitivity is anticipated.

\section{(iii) Pulse Height and Gain Calibration}

The good agreement between the shapes of the measured pulse height spectrum (figure 3 ) and the Montecarlo model spectrum gives one confidence in the energy calibration derived from it and the existence of a peak in the experimental spectrum provides a very useful monitor of the counter stability as well as a gain calibration.

The Montecarlo model predicts a small peak in the energy spectrum at the full energy deposit of $790 \mathrm{keV}$. This is not featured in the experimental spectrum and a straggle of pulses continues beyond the nominal $\mathrm{Q}$ of the reaction. This is a consequence of the high energy gain tail generated by the drift field configuration in the MSGD. In conditions with a small drift gap and high drift field a significant portion (up to 10\%) of the electrons collected onto the anode strips experience a significantly higher gain which washes out any small peaks lying on the top edge of an intense distribution.

The MSGD gain curves presented in figure 4 are just as one would expect in hyperbaric operation. The maximum gain of 200 observed at both operating pressures seems low but is in fact quite satisfactory for operating the electronics. The pulse height spectra peaks at this gain are at $7 \times 10^{5}$ electrons (3bar) and $1.4 \times 10^{6}$ electrons (5.4bar) respectively giving a good signal to noise ratio at the discriminators.

\section{(iv) Spatial Resolution}

Characterisation of the spatial resolution achievable with the MSGD was the principal focus of the present tests. Thus the sequence of measurements of the PSF at different LLD settings was repeated with the detector moved $4.5 \mathrm{~mm}$ across the $0.25 \mathrm{~mm}$ wide slit (Figure 5). In one case the slit was aligned with a detector strip and in other it split between two strips. The spatial resolution fit parameters and the sensitivity agreed perfectly (within the statistical errors).

The spatial fit parameters shown in figure 7 show that at maximum sensitivity $(\mathrm{LLD}=44.5 \mathrm{keV})$ the counts in the PSF are divided approximately equally between the two gaussian contributions with a narrow peak with $\sigma_{1}=0.33 \mathrm{~mm}$ and a wider peak with $\sigma_{2}=1.2 \mathrm{~mm}$. At the other extreme (LLD=402keV) there is $60 \%$ in $\sigma_{1}=0.11 \mathrm{~mm}$ and $40 \%$ in $\sigma_{2}=0.49 \mathrm{~mm}$. The width of the collimating slit is significant in the latter case $(\sigma=$ 
$0.25 / \sqrt{ } 12=0.072 \mathrm{~mm}$ ) and subtracting in quadrature indicates an intrinsic detector resolution of $0.083 \mathrm{~mm}$.

At low LLD settings the Montecarlo model gives good fits to the bi-gaussian form of the PSF with good agreement on $\sigma_{1}$ but with a tendency to underestimate $\sigma_{2}$. As the LLD rises (particularly in simulations at lower pressures) while the model predicts the core gaussian shape well, the wider component is significantly different. The shoulders which appear instead of the outer gaussian (figure 10) are clearly due to the bragg peak of tritons stopping in the adjacent counter strip. However, in the experimental situation this effect is not seen due to various dispersive effects which are not modelled in the Montecarlo. Low level dispersions on the scale of $1 \mathrm{~mm}$ will arise from neutron scattering in the aluminium window and from penumbral effects of scattering in the boron carbide collimator. The pulse height dispersive effect noted in figure 3 operates on charge generated near the strip boundary and will also help to increase $\sigma_{2}$. It is thus not surprising that the model underestimates the fraction of the signal and the magnitude of $\sigma_{2}$ : at $L L D=135 \mathrm{keV}$, Fraction 2 is modelled to be $25 \%$ while $41 \%$ is observed and $\sigma_{2}$ is modelled as $0.75 \mathrm{~mm}$ and observed as $1.1 \mathrm{~mm}$.

\section{(v) Sensitivity}

The maximum spatial resolution of the detector is attained with a loss of a factor of 22 in counting rate (sensitivity). For the imaging of features of size $>\sigma_{2}$ this decline with LLD setting is the parameter of interest (and clearly if high spatial resolution is not needed then the LLD should be kept low); however, for features of size $\leq \sigma_{1}$ the important parameter is the normalised amplitude of the narrow gaussian. This is plotted in figure 8 as $\mathrm{S}$ (sensitivity)*Fraction ${ }_{1} / \sigma_{1}$. This parameter is proportional to the system's imaging ability for small features and, as figure 8 shows this declines only by a factor of 6 over the full range of LLD.

Figure 8 also shows the true detection efficiency of the detector (i.e. the probability that there is at least one count per neutron) as modelled by the Montecarlo. This parameter declines less steeply than the sensitivity. The ratio between the sensitivity and the detection efficiency simply represents the number of hits per neutron (multiplicity). At high LLD settings the multiplicity asymptotes to unity.

\section{(vi) Spatial Resolution with 5bar Gas Filling}

The main benefits of doubling the gas pressure in the counter are the doubling of the sensitivity and the pulse height. Unfortunately there is little benefit in respect of the ultimate spatial resolution. As figure 9 shows the area-normalised PSF improves (as expected) with increasing LLD. However, this improvement results almost entirely from the fact that Fraction ${ }_{1}$ increases from $38 \%$ to $83 \%$ while $\sigma_{1}$ and $\sigma_{2}$ remain substantially constant at $0.21 \mathrm{~mm}$ and $0.75 \mathrm{~mm}$ respectively.

The Montecarlo model confirms this counterintuitive performance as Figure 10 shows. As with the 3 bar results the model is seen to underestimate the wider gaussian. The marked asymmetry seen in the experimental PSF tail would seem to point to an effect of the experimental set-up rather than an intrinsic detector feature. 


\section{FURTHER DEVELOPMENTS}

The present tests have demonstrated that the MSGD imaging beam monitor can deliver spatial resolution in one dimension of the order of $0.1 \mathrm{~mm}-0.3 \mathrm{~mm}(\sigma)$ with a sensitivity in the region of $1 \%$. Work is now in hand to develop a two dimensional version with comparable performance. Enough electronics are available to realise a detector with $48 \times 48$ channels of readout.

\section{REFERENCES}

1. A Oed, Nucl Instr \& Meth A263 (1988) 351-359

A Oed et.al., A large, high counting rate, one-dimensional, position sensitive ndetector: the D20 banana, submitted to Physica B: Physics of Condensed Matter.

2. J E Bateman, J F Connolly, R Stephenson, M Edwards and J C Thompson, Nucl. Instr. and Meth. A348 (1994) 372

3. J E Bateman, N J Rhodes and R Stephenson, An imaging neutron beam monitor using gas microstrip technology, Rutherford Appleton Laboratory, RAL-TR-97-021.

4. J E Bateman, N J Rhodes and R Stephenson, The performance of gas microstrip and gas pixel detectors for use on ISIS beam lines - a Montecarlo study, Rutherford Appleton Laboratory, RAL-TR-98-024.

\section{FIGURE CAPTIONS}

Figure 1 The profile of the ROTAX neutron beam as imaged by the MSGD beam monitor (3bar gas filling, $\mathrm{V}_{\mathrm{c}}=1000 \mathrm{~V}, \mathrm{LLD}=44.5 \mathrm{keV}$ )

Figure 2 The flight time spectra of the MSGD beam monitor in the ROTAX neutron beam with the PRISMA beam monitor for comparison.

Figure 3 The pulse height spectrum measured from the MSGD beam monitor under neutron irradiation from a $0.25 \mathrm{~mm}$ wide slit (5.4bar gas filling, $\left.\mathrm{V}_{\mathrm{c}}=1199 \mathrm{~V}\right)$. The pulse height spectrum produced by the Montecarlo model is peak-normalised to the experimental distribution.

Figure 4 The MSGD gain curves derived from the pulse height spectra by fitting to the Montecarlo spectra and assuming an energy per ion pair in the gas of $28 \mathrm{eV}$.

Figure 5 MSGD images of the $0.25 \mathrm{~mm}$ wide neutron beam with the detector moved $4.5 \mathrm{~mm}$ between exposures. 
Figure 6. The area-normalised point spread functions (PSF) of the images of the $0.25 \mathrm{~mm}$ wide neutron beam as a function of the energy discriminator LLD.

Figure $7 \quad$ The sensitivity and bi-gaussian fit parameters of the experimental PSFs shown in figure 6 (as a function of the discriminator LLD).

Figure 8 The measured sensitivity and the Montecarlo model values are normalised together at $\mathrm{LLD}=50 \mathrm{keV}$ and plotted. Also plotted is the neutron detection efficiency and a parameter $\left(\mathrm{S}^{*}\right.$ Fraction $\left._{1} / \sigma_{1}\right)$ which is proportional to the absolute (i.e. per incident neutron) amplitude of the narrow gaussian.

Figure 9 The area-normalised PSFs obtained from the $0.25 \mathrm{~mm}$ slit when the MSGD was filled to 5.4bar (as a function of the LLD).

Figure 10 A comparison of the measured PSFs in the MSGD with a 3bar and 5.4bar filling with the corresponding PSFs predicted by the Montecarlo model. 
FIGURE 1

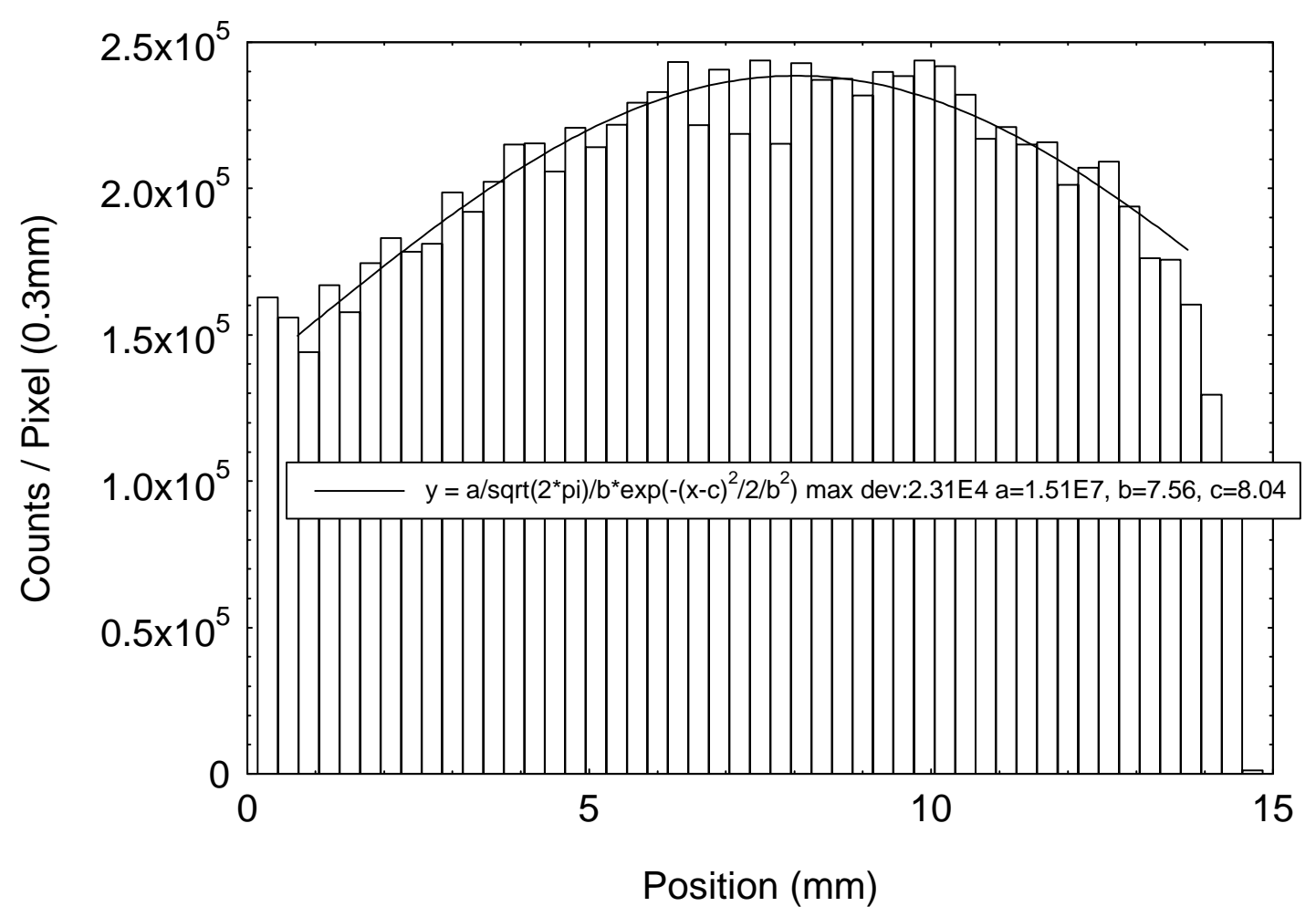

FIGURE 2

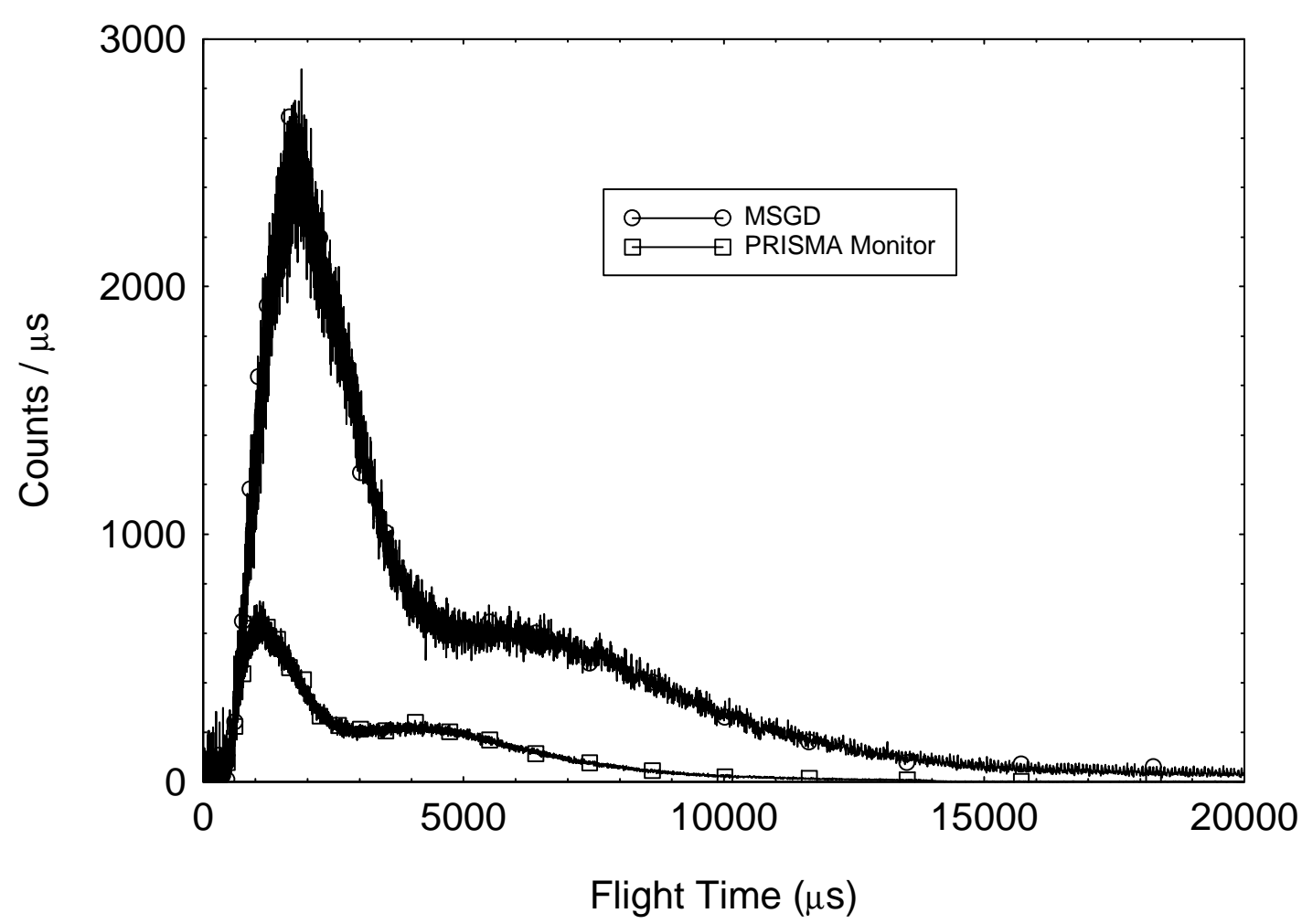


FIGURE 3
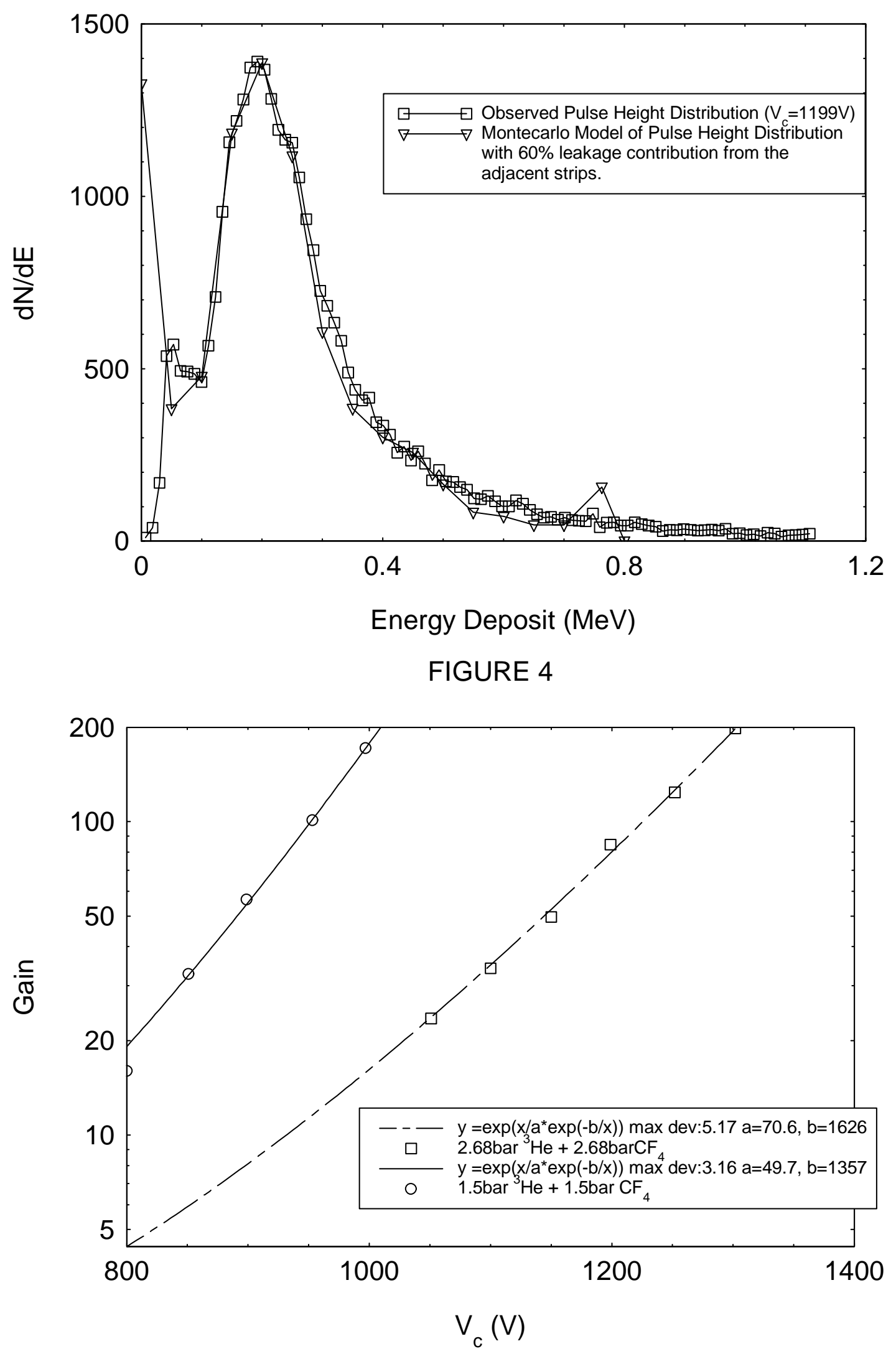
FIGURE 5

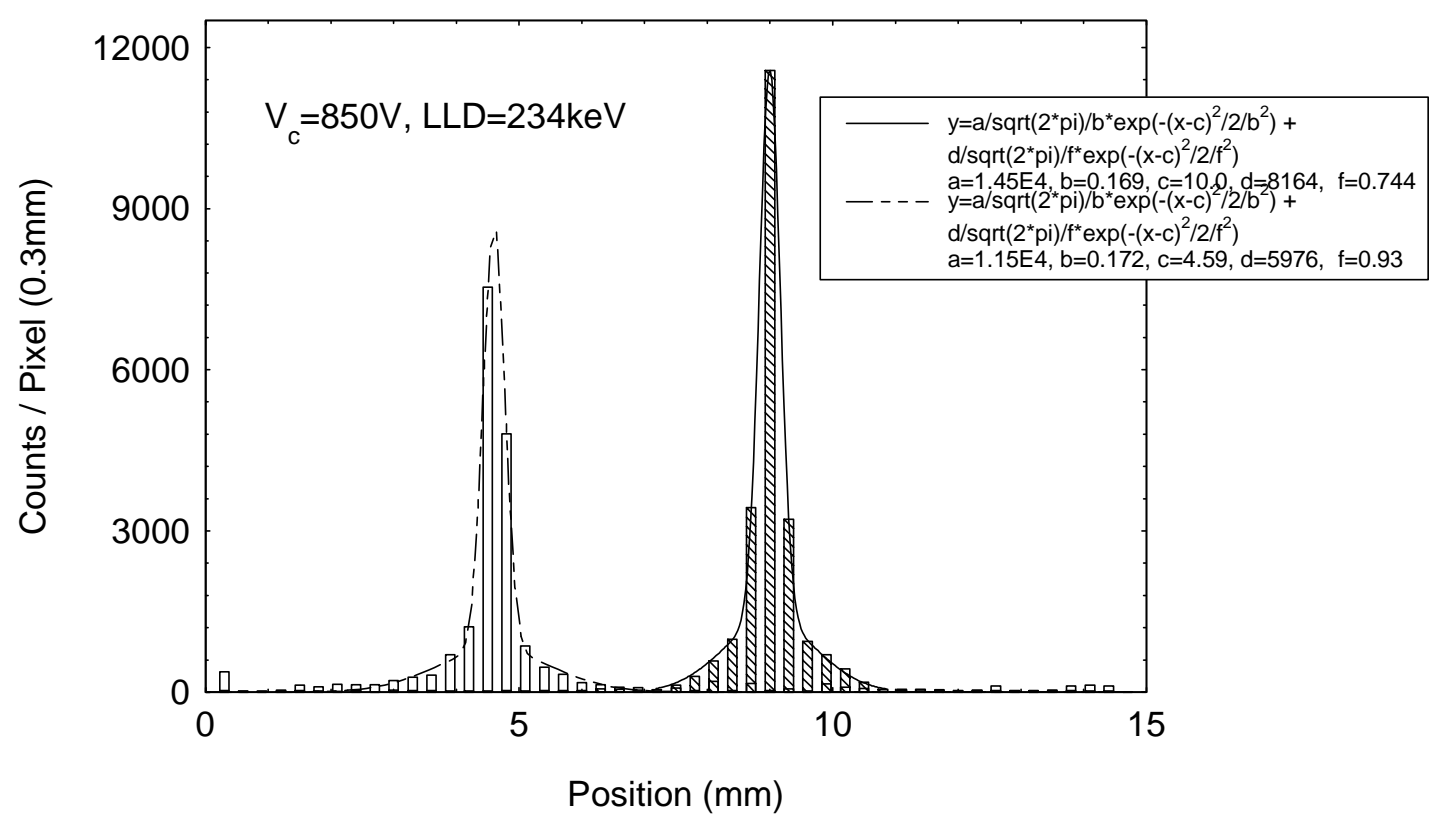

FIGURE 6

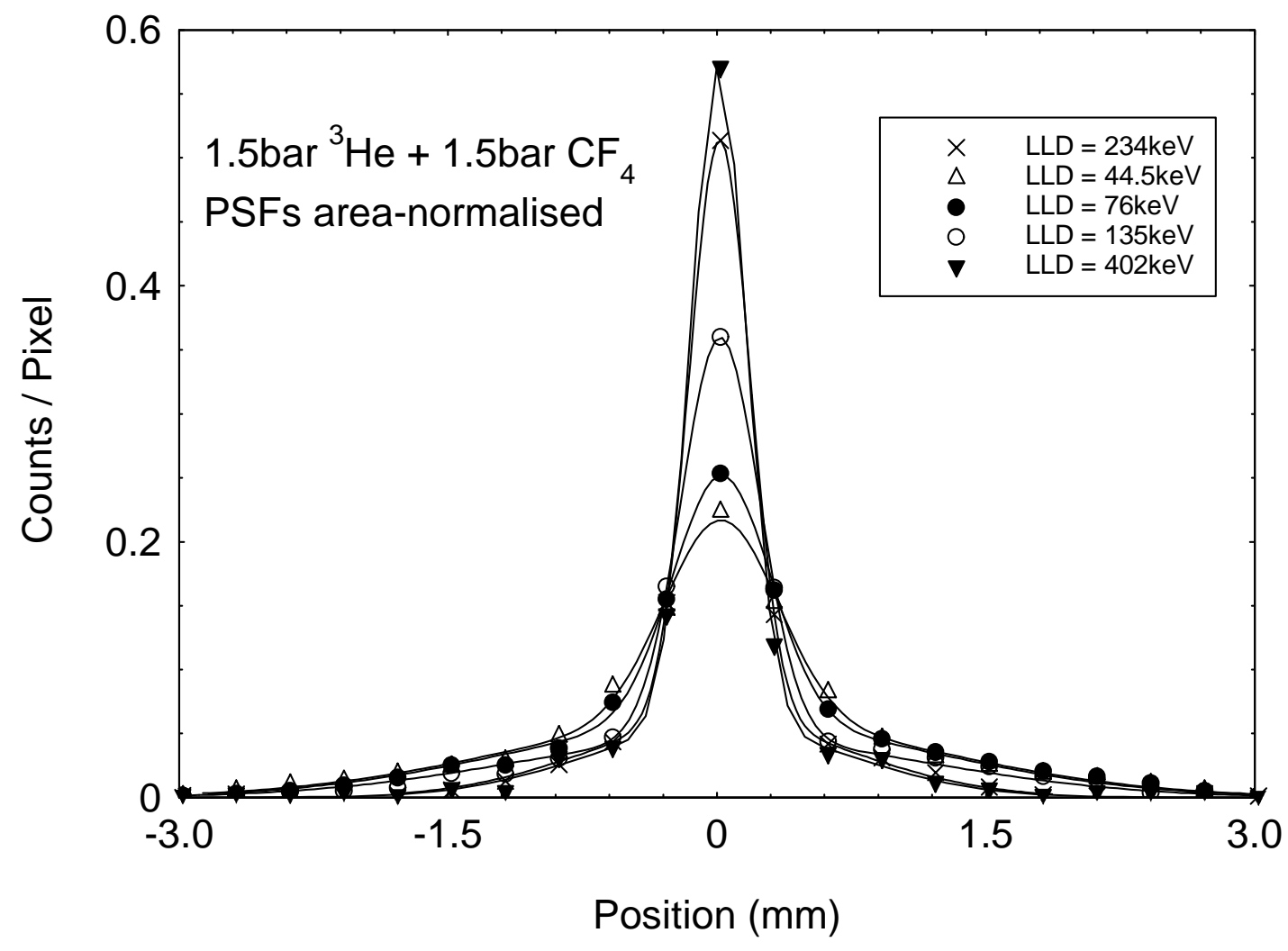


FIGURE 7

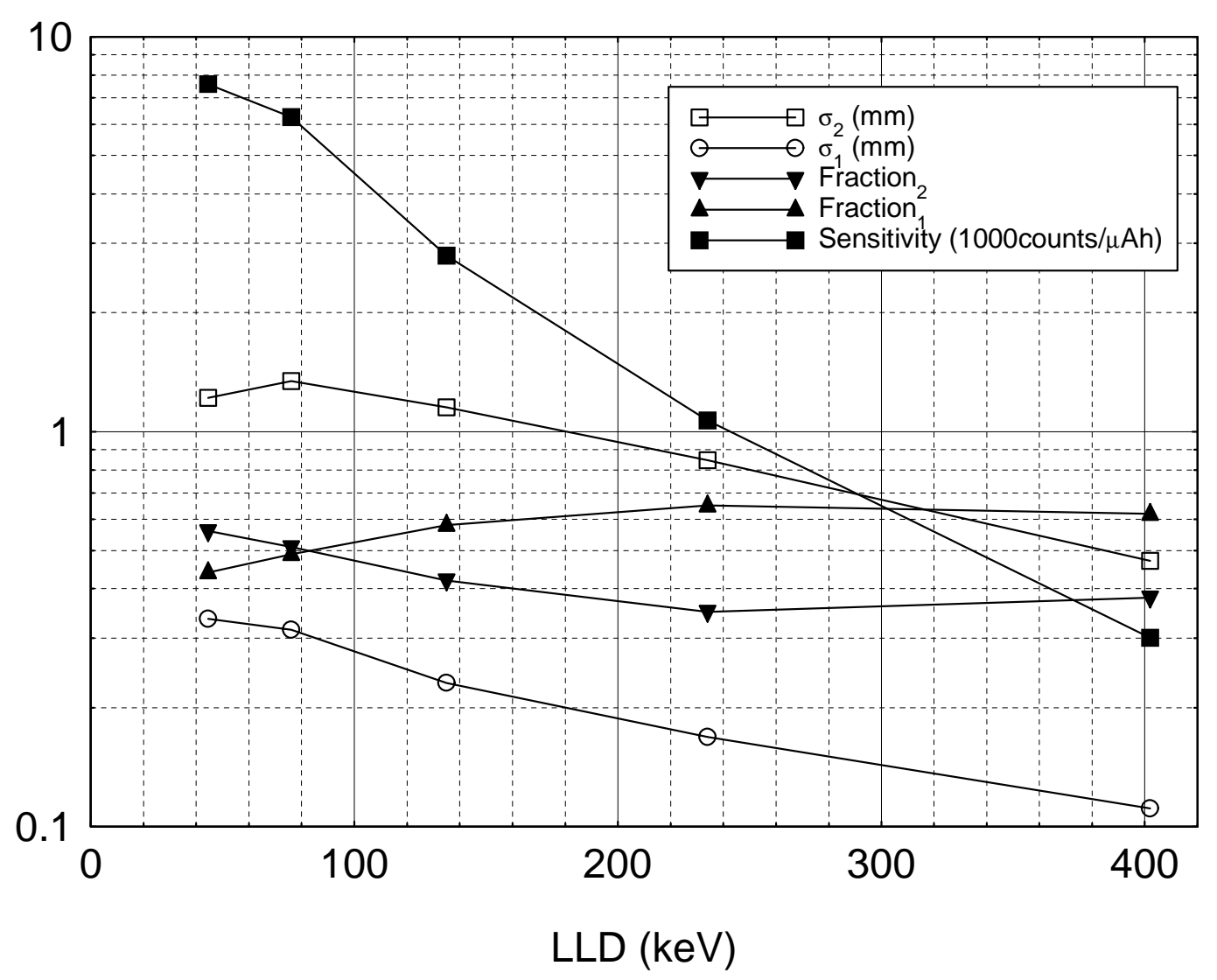

FIGURE 8

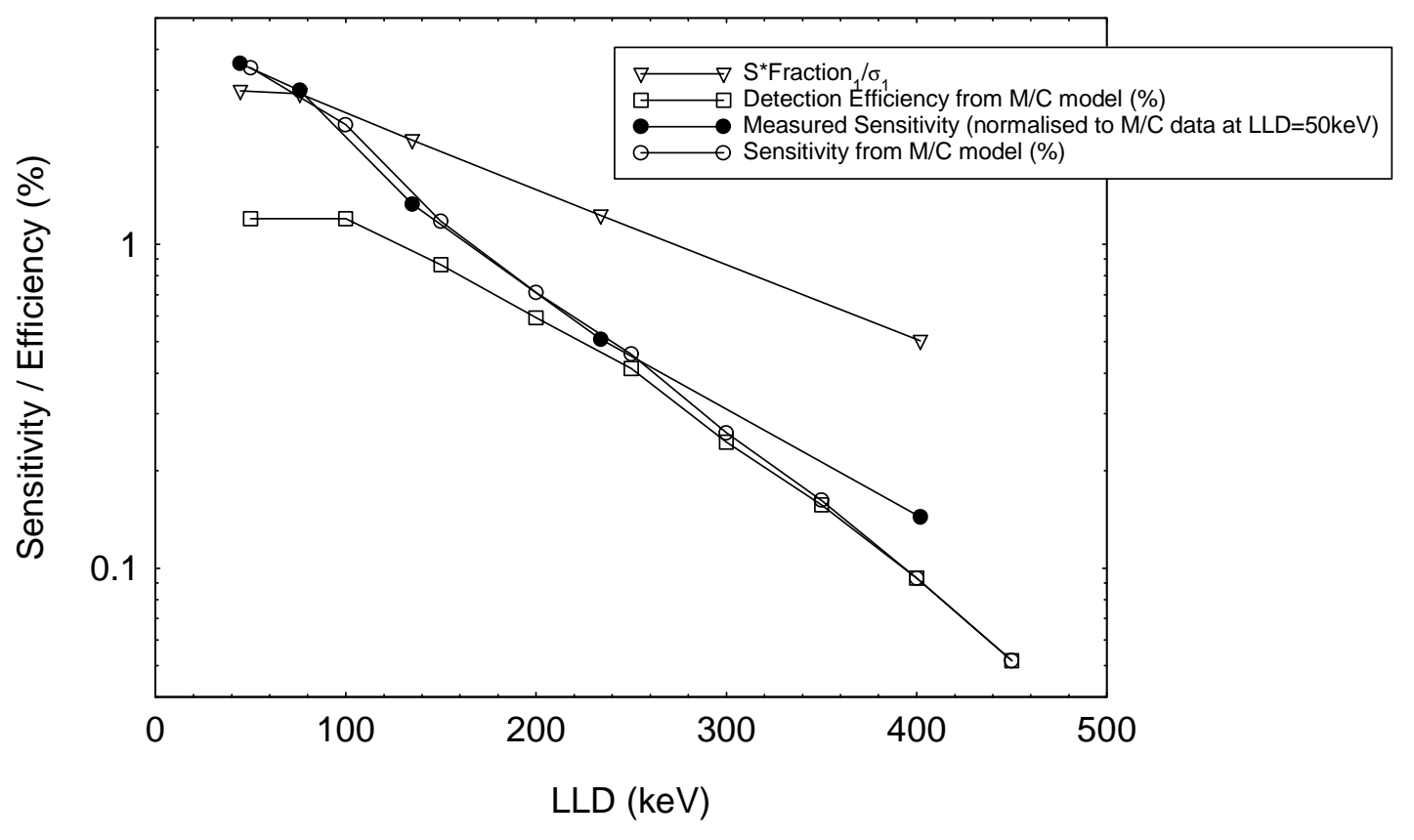


FIGURE 9

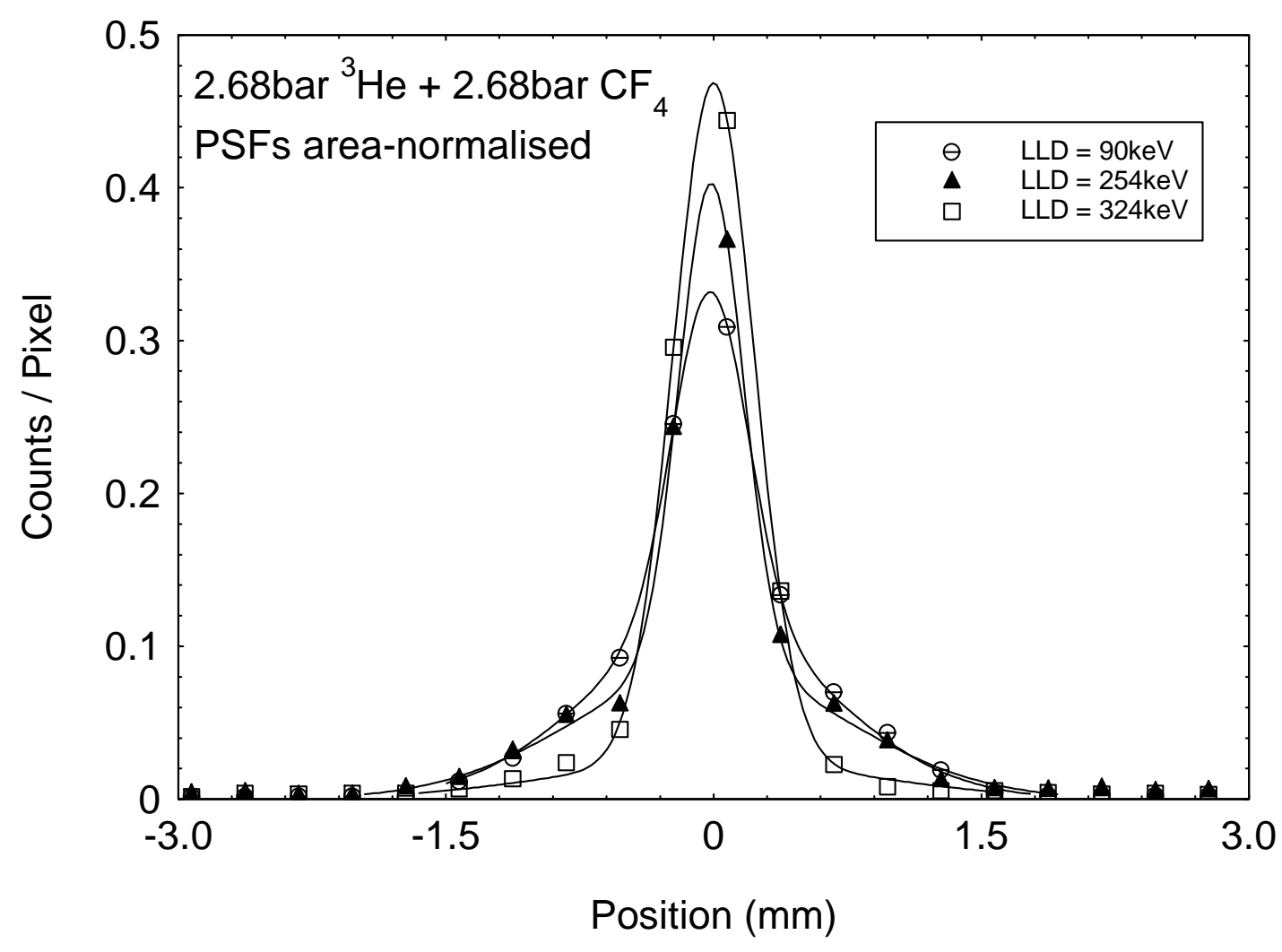

FIGURE 10

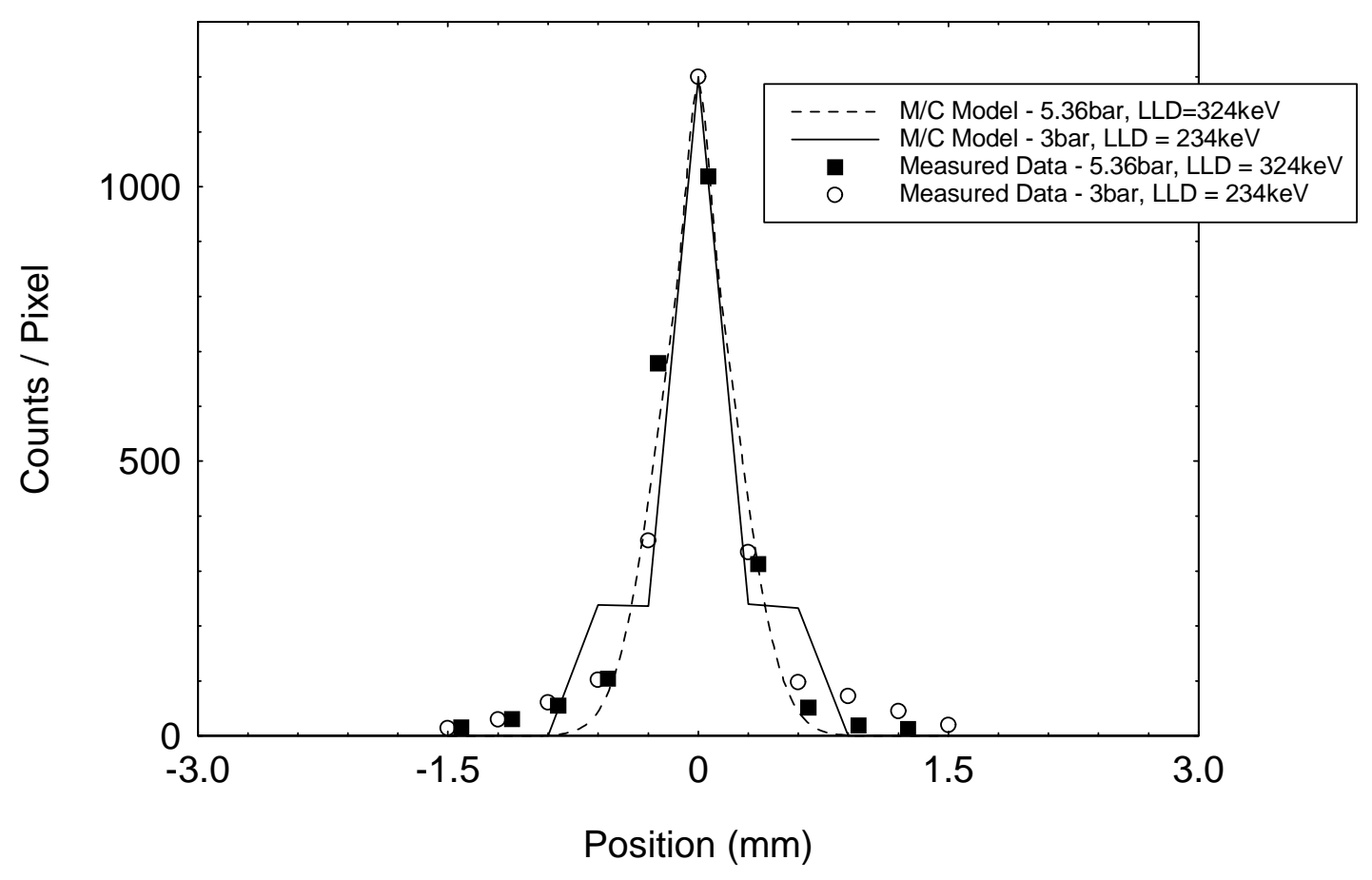

